the potential to improve our practice of prescribing benzodiazepine and Z-drugs, it was decided to evaluate current use.

Method. The NICE guidelines were consulted, and we retrospectively reviewed the use of these agents from mid-January to the end of May 2020. Demographic variables included age, gender, and county. Patients were stratified into three groups, the benzodiazepine group, the Z-drugs group, and the combined benzodiazepine and Z-drugs group. In each group therapeutic variables were recorded including the medication type, dose, frequency, prescriber, and duration of treatment. Other variables included psychiatric diagnoses, length of inpatient admission, status on admission, and recommendations on discharge

Result. There were 101admissions during that period, and 74 of them were prescribed these agents $(n=74 ; 73.3 \%)$. Fifty one ( $n$ $=51 ; 68.9 \%)$ received benzodiazepines only, twenty-three $(\mathrm{n}=$ $23 ; 31.1 \%)$ were prescribed Z-drugs, and twelve $(n=12 ; 16.2 \%)$ received both benzodiazepines and Z-drugs. Forty two patients $(\mathrm{n}=42 ; 56.8 \%)$ were commenced on hypnotics in the APU, 23 patients $(n=23 ; 31.1 \%)$ already received hypnotics from the CMHTs, and the rest were prescribed by both. Thirty two patients $(\mathrm{n}=32 ; 43.2 \%)$ were discharged on hypnotics. Patients admitted involuntarily and female patients had longer admissions (mean of $16.62 \pm 3.26$ days and $16.16 \pm 2.89$ days respectively). Schizophrenia and $\mathrm{BPAD}$ were the commonest diagnoses.

Conclusion. It appears that large amounts of these agents are used in the Acute Hospital Setting which is not overly surprising given the severity of illness and clinical indications however improved awareness could still lead to more appropriate and hopefully reduced use. We therefore recommend:

A formal audit including appropriate interventions i.e., educate staff and patients, highlight guidelines, and review subsequent practice.

Train staff in safer prescribing practices including prn rather than regular use if appropriate.

Regularly review discharge prescriptions indicating recommended duration of use.

\section{Root causes of deaths by suicide amongst patients under the care of a mental health trust: a thematic analysis}

\author{
Dhruba Bagchi ${ }^{\star}$, Kerry Webb ${ }^{2}$ and Opeyemi Odejimi ${ }^{2}$ \\ ${ }^{1}$ Birmingham Community Healthcare NHS Foundation Trust and \\ ${ }^{2}$ Birmingham and Solihull Mental Health NHS Foundation Trust \\ ${ }^{\star}$ Corresponding author.
}

\section{doi: 10.1192/bjo.2021.819}

Aims. This study explores common themes emerging from root causes of Serious Incident (SI) reports of mental health service users who died by suicide under the care of a mental health trust. Background. Suicide is a global health problem. It is estimated every year about 800,000 people die by suicide worldwide. Previously, the United Kingdom (UK) reported a significant reduction. However, the latest report in 2018 indicated a marked increase. Furthermore, $28 \%$ of people who died by suicide in the UK were under the care of mental health service 12 months prior to their death. The causes of suicide are not usually straightforward, but sometimes could be preventable. Thus exploring the root causes is a step in the right direction to preventing this global problem.

Method. Thematic analysis was carried to identify themes emerging from the Root Causes (RCs) within the Serious Incident (SI) reports of patients who died by suicide while under the care of the Trust between January 1st, 2017 and July 31st, 2018. Over the 18 month period, there were 71 deaths, of which 36 were ruled as suicide by the coroner. A further 16 were considered by the review team as possible suicide and were therefore included to increase the scope of learning. This review is therefore based on 48 cases. Result. Three main themes emerged from this study. They are patient, professional and organisational factors. Majority of the death were patient related factors, particularly exacerbation of patient's mental health condition. Furthermore, the most frequently occurring professional and organisational factor were issues around patient risk assessment and management and inadequate psychiatric bed respectively.

Conclusion. The findings of this study have helped gained an understanding of the perceived causes of death of patient who died by suicide. It is hoped that this will in turn influence the manner in which, decisions, policies and resource allocation are carried out to further prevent and reduce the incidence of suicide, particularly amongst mental health patients.

\section{Pharmacological treatment of post-traumatic stress disorder- an audit of Cardiff Health access practice using a pharmacological prescribing algorithm}

Amy Baker* and Jonathan Bisson

Cardiff University

${ }^{\star}$ Corresponding author.

doi: 10.1192/bjo.2021.820

Background. Post-Traumatic Stress Disorder (PTSD) is a mental health disorder characterised by symptoms of re-experiencing, avoidance and hyperarousal that may develop after exposure to a traumatising event. The prevalence of PTSD within the refugee population is ten times higher than in the general population. This audit was carried out in Cardiff Health Access Practice (CHAP) which is the main provider of primary health care for refugees and asylum seekers who are sent to Cardiff. The main objective of this audit was to evaluate current PTSD prescribing practice for patients presenting to Cardiff Health Access Practice (CHAP) against a pharmacological prescribing algorithm which has been developed for the Cardiff and Vale Traumatic Stress Service based on NICE and International Society for Traumatic Stress Studies guidelines

Method. A retrospective audit of patients with PTSD seen in the last 12 months at CHAP. Data were collected from patient notes and information on age, sex, trauma, comorbidities and medication dose was collated and analysed using SPSS statistics.

Result. 130 patients with PTSD were identified and their medications assessed for the audit. The mean age of these patients was 33 years and there was a $1.5: 1$ male to female ratio. Of the 130 patients only 10 were initiated on a first line medication, 117 were started on a fourth line medication. No patients were prescribed either the second- or third-line medications.

Conclusion. The low rates of compliance with the All Wales Pharmacological PTSD pharmacological prescribing algorithm are disappointing although not unexpected as it has yet to be fully introduced to the service. Following discussion of the results and teaching about the algorithm with clinicians in Cardiff Health Access Practice rates of evidence-based prescribing should improve. This audit focuses on a patient group (refugee and asylum seekers) which has been identified as a priority group by the Welsh Government. Through further implementation of this algorithm there should be improved evidence-based prescribing and continuity of care for refugees 